\title{
Analysis of SEER Adenosquamous Carcinoma Data to Identify Cause Specific Survival Predictors and Socioeconomic Disparities
}

\section{Rex Cheung}

\begin{abstract}
Background: This study used receiver operating characteristic curve to analyze Surveillance, Epidemiology and End Results (SEER) adenosquamous carcinoma data to identify predictive models and potential disparities in outcome. Materials and Methods: This study analyzed socio-economic, staging and treatment factors available in the SEER database for adenosquamous carcinoma. For the risk modeling, each factor was fitted by a generalized linear model to predict the cause specific survival. An area under the receiver operating characteristic curve (ROC) was computed. Similar strata were combined to construct the most parsimonious models. Results: A total of 20,712 patients diagnosed from 1973 to 2009 were included in this study. The mean follow up time (S.D.) was 54.2 (78.4) months. Some $2 / 3$ of the patients were female. The mean (S.D.) age was 63 (13.8) years. SEER stage was the most predictive factor of outcome (ROC area of 0.71$) .13 .9 \%$ of the patients were un-staged and had risk of cause specific death of $61.3 \%$ that was higher than the $45.3 \%$ risk for the regional disease and lower than the $70.3 \%$ for metastatic disease. Sex, site, radiotherapy, and surgery had ROC areas of about 0.55-0.65. Rural residence and race contributed to socioeconomic disparity for treatment outcome. Radiotherapy was underused even with localized and regional stages when the intent was curative. This under use was most pronounced in older patients. Conclusions: Anatomic stage was predictive and useful in treatment selection. Under-staging may have contributed to poor outcome.
\end{abstract}

Keywords: Adenosquamous carcinoma - radiotherapy - SEER registry - under usage - cause specific survival

Asian Pac J Cancer Prev, 17 (1), 347-352

\section{Introduction}

SEER registry has massive amount of data available for analysis, however, manipulating this data pipeline could be challenging. SEER Clinical Outcome Prediction Expert (SCOPE) (Cheung, 2014c; Cheung, 2014a; Cheung, 2014d; Cheung, 2014b) was used mine SEER data and construct accurate and efficient prediction models (Cheung et al., 2001a; Cheung et al., 2001b). The data were obtained from SEER 18 database. SEER is a public use database that can be used for analysis with no internal review board approval needed. SEER*Stat (http://seer. cancer.gov/seerstat/) was used for listing the cases. The filter used was: Site and Morphology.ICD-O-3 Hist/behav, malignant $=$ '8560/3: Adenosquamous carcinoma'. This study explored a long list of socio-economic, staging and treatment factors that were available in the SEER database (Cheung, 2014a; 2014b; 2014c; 2014b; 2014e; Cheung, 2015a; 2015b; Cheung, 2015 (In press)).

The codes of SCOPE are posted on Matlab Central (www.mathworks.com). SCOPE has a number of utility programs that are adapted to handle the large SEER data pipeline. All statistics and programming were performed in Matlab (www.mathworks.com) (Cheung, 2014a; 2014b; 2014c; 2014b; 2014e; Cheung, 2015a; 2015b; Cheung, 2015 (In press)). The areas under the receiver operating characteristic curve (ROC) were computed (Cheung, 2014a; 2014b; 2014c; 2014b; 2014e; Cheung, 2015a; 2015b; Cheung, 2015 (In press)). Similar strata were fused to make more efficient models if the ROC performance did not degrade (Cheung et al., 2001a; Cheung et al., 2001b). In addition, it also implemented binary fusion and optimization to streamline the risk stratification by combining risk strata when possible (Cheung, 2014a; 2014b; 2014c; 2014b; 2014e; Cheung, 2015a; 2015b; Cheung, 2015 (In press)). SCOPE provides SEER-adapted programs for user friendly exploratory studies, univariate recoding and parsing (Cheung, 2014a; 2014b; 2014c; 2014b; 2014e; Cheung, 2015a; 2015b; Cheung, 2015 (In press)).

\section{Materials and Methods}

SEER registry has massive amount of data available for analysis, however, manipulating this data pipeline could be challenging. SEER Clinical Outcome Prediction Expert (SCOPE) (Cheung, 2014a; 2014b; 2014c; 2014b) was used mine SEER data and construct accurate and 
Table 1. The Risk Models Include the Sociodemographic, Tumor and Treatment Factors for Adenosquamous Carcinoma

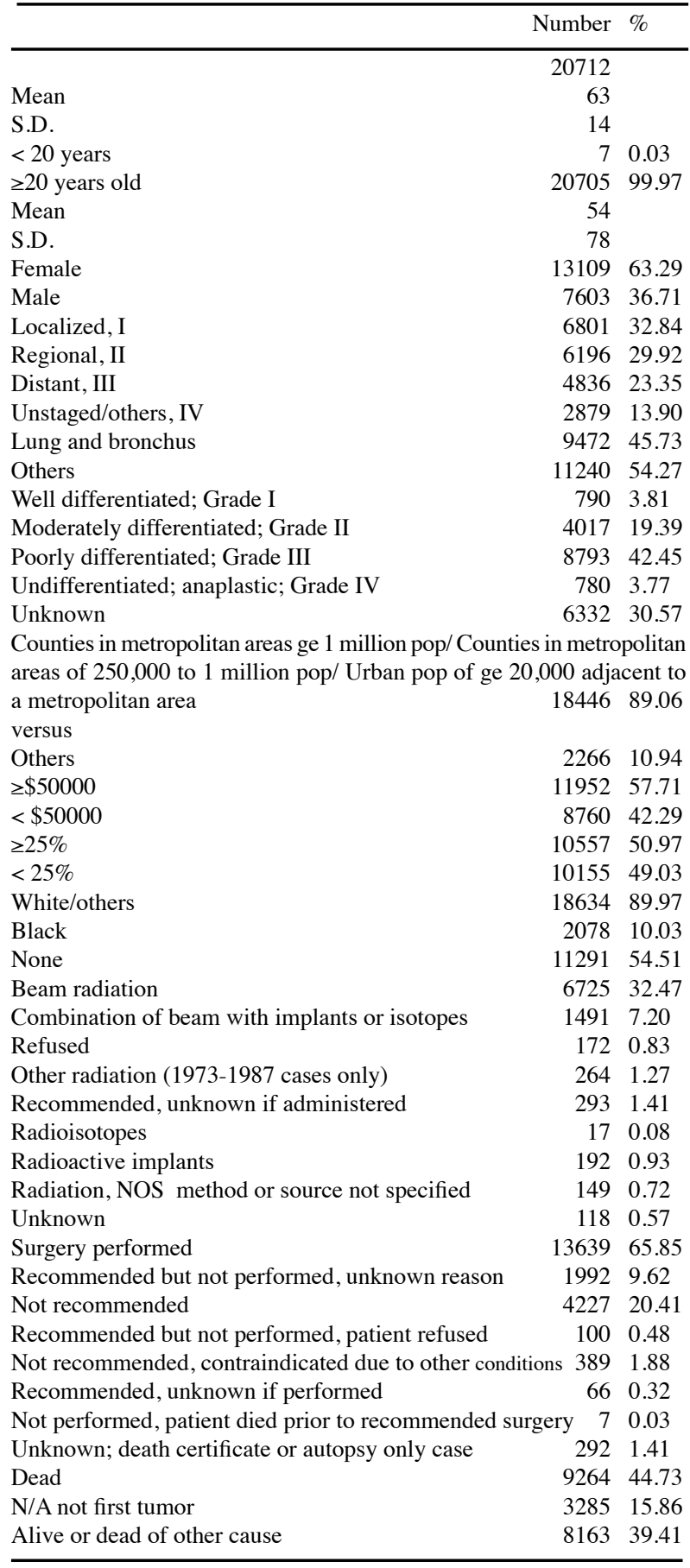

efficient prediction models (Cheung et al., 2001a; 2001b). The data were obtained from SEER 18 database. SEER is a public use database that can be used for analysis with no internal review board approval needed. SEER*Stat (http:// seer.cancer.gov/seerstat/) was used for listing the cases. The filter used was: Site and Morphology.ICD-O-3 Hist/ behav, malignant $=$ '8560/3: Adenosquamous carcinoma'. This study explored a long list of socio-economic, staging and treatment factors that were available in the SEER database (Cheung, 2014a; 2014b; 2014c; 2014b; 2014e; Cheung, 2015a; 2015b; Cheung, 2015 (In press)).

The codes of SCOPE are posted on Matlab Central (www.mathworks.com). SCOPE has a number of utility programs that are adapted to handle the large SEER data pipeline. All statistics and programming were performed in Matlab (www.mathworks.com) (Cheung, 2014a; 2014b; 2014c; 2014b; 2014e; Cheung, 2015a; 2015b; Cheung, 2015 (In press)). The areas under the receiver operating characteristic curve (ROC) were computed (Cheung, 2014a; 2014b; 2014c; 2014b; 2014e; Cheung, 2015a; 2015b; Cheung, 2015 (In press)). Similar strata were fused to make more efficient models if the ROC performance did not degrade (Cheung et al., 2001a; Cheung et al., 2001b). In addition, it also implemented binary fusion and optimization to streamline the risk stratification by combining risk strata when possible (Cheung, 2014a; 2014b; 2014c; 2014b; 2014e; Cheung, 2015a; 2015b; Cheung, 2015 (In press)). SCOPE provides SEER-adapted programs for user friendly exploratory studies, univariate recoding and parsing (Cheung, 2014a; 2014b; 2014c; 2014b; 2014e; Cheung, 2015a; 2015b; Cheung, 2015 (In press)).

\section{Results}

There were 20712 patients included in this study (Table 1). The follow up (S.D.) was 54.2 (78.4) months. $64 \%$ of the patients were female. The mean (S.D.) age was 63 (13.8) years. There were $60 \%$ adenosquamous carcinoma patients listed from SEER database were adults. There were 7 patients younger than 20 years old in the SEER data, and it was a poor prognostic factor (Table 1 and Table 2). There is a significant female to male difference in risk of cause specific death (Table 2) favoring the female sex. $46 \%$ of the patients had lung cancers. Uterus and uterine cervix were also the common anatomic sites (Table 3). 30.6\% of the tumors were not graded. Unknown grade has the highest risk of cause specific death at $51.8 \%$. SEER stage model (localized, regional, distant, un-staged/others) was the most predictive model (ROC area or 0.71). A 4-tiered staging model was optimized to a 3-tiered model (with a ROC area of 0.67) by SCOPE (Table 1). ROC areas were used to optimize the risk models. For example, the SEER staging could be slimmed down to 3-tiered structure while not abandoning the poor (Table 1, 2 and 3). Among the socioeconomic factors studies, African American patients had 53.8\% risk of death compared with $43.7 \%$ of others. However, this level of difference increased the ROC area mildly to 0.52 (Table 1). A rural residence and living a cosmopolitan area have respectively $48.7 \%$ and $44.2 \%$ risk of cause specific death (Table1, 2 and 3).

There is about $44.73 \%$ overall risk of adenosquamous carcinoma death for patients listed in SEER. The risks were $19.1 \%$ and $45.3 \%$ for localized and regional adenosquamous carcinoma respectively (Table 2). Age older than 20 years old did correlate with higher percentage mortality during this study period from 1973 to 2009 (Table 1 and Table 2). RT with external beam was associated with $54.5 \%$ risk of death, and $32.5 \%$ risk of death without RT (Table 2). Patients had surgery had $34 \%$ risk of death, $66 \%$ risk of death among patients who did not have surgery. 
DOI:http://dx.doi.org/10.7314/APJCP.2016.17.1.347 Analysis of SEER Adenosquamous Carcinoma Data for Cause Specific Survival Predictors and Socioeconomic Disparities

Table 1. The Risk Models Include the Socio-demographic, Tumor and Treatment Factors for Adenosquamous Carcinoma (Continue)



\section{Discussion}

This study is interested in constructing models that will aid patient and treatment selection for adenosquamous carcinoma cancer patients. To that end, this study examined the ROC models (Hanley and McNeil, 1982) of a long list of potential explanatory factors (Table 1). ROC models take into account both sensitivity and specificity of the prediction. Ideal model would have a ROC area of 1 and a random model is expected to have an area of
0.5 (Hanley and McNeil, 1982; Cheung, 2014c; Cheung, 2014a; Cheung, 2014d; Cheung, 2014b; Cheung, 2014e; Cheung, 2015b; Cheung, 2015a; Cheung, 2015 (In press)). For example, a clinical ROC model can be used to predict if a patient receiving the recommended treatment will die from the disease. SEER stage in order to be consistent over decades, it abstracts the staging into simple but important stages for cancer progression: localized, regional and distant. Stage was the most predictive of patient outcome (Table 1). Stage has ROC of 0.71 was higher than the 


\section{Rex Cheung}

0.65 of surgery. Thus complete staging is important in this disease and it may improve patient selection and council.

After binary fusion by SCOPE (Table 1), the 4 tiered stage was reduced to a 3 tiered grade based on ROC area calculations (Table 1). Un-staged grade was associated with high risk of cause specific death (Table 2). However, there is no a priori reason to put it between localized and distant. Thus it was left as a high risk factor. The solution to the uncertainty of placement of these cases is to complete the staging (Cheung, 2014c; Cheung, 2014a; Cheung, 2014d; Cheung, 2014b). The binary fusion was performed to demonstrate how a complex predictive model could be numerically optimized to a much simpler model that may also be useful (Cheung, 2014c; Cheung, 2014a; Cheung, 2014d; Cheung, 2014b).

When there are competing prediction or prognostic models, the most efficient (i.e. the simplest) model is thought to prevail (D'Amico et al., 1998). This has an information theoretic under-pinning (Cheung, 2014c; Cheung, 2014a; Cheung, 2014d; Cheung, 2014b). For practical purposes, simpler models require fewer patients for a randomized trials because fewer risk strata need to be balanced using epidemiology data (Cheung, 2014a; 2014b; 2014c; 2014b; 2014e; Cheung, 2015a; 2015b; Cheung, 2015 (In press)). In the clinic, simpler models are easier to use. SCOPE streamlined ROC models by binary fusion (Table 1). Two adjacent strata were tested iteratively to see if they could be combined without sacrificing the higher predictive power usually belong to the more complex models (Cheung, 2014c; Cheung, 2014a; Cheung, 2014d;
Cheung, 2014b). This study has shown that SCOPE can build efficient and accurate prediction models (Cheung, 2014c; Cheung, 2014a; Cheung, 2014d; Cheung, 2014b).

For optimized stage model (Table 1), the ROC area of 0.67 was modestly more than that of surgery. For a point of reference, using we computed the prostate risk model was 0.75 in its accuracy of predicting biochemical failure(Cheung et al., 2001a; Cheung et al., 2001b). Low ROC areas imply the information content (i.e. the staging accuracy) of the models may be limited. It is consistent with the fact that most patients did not have complete grading or staging (Table 2). This is an area of improvement. It may be a consequence of having a better guidance model in treatment and patient selection.

Adenosquamous carcinoma is an aggressive disease, there was a $19 \%$ risk of adenosquamous carcinoma death (Table 2) despite treatments even for early stage cancer.

In conclusion, this study has identified the staging models are the most prognostic of treatment outcomes of adenosquamous cancer patients. The high under-staging rates may have prevented patients from selecting definitive local therapy and may have contributed to the poor outcome in these patients with this aggressive disease.

\section{References}

Adegoke O, Kulasingam S, Virnig B (2012). Cervical cancer trends in the United States: a 35-year population-based analysis. $J$ Womens Health.

Bhatia S (2011). Disparities in cancer outcomes: lessons learned

Table 2. Risk of Cause Specific Mortality (\%) Associated with Different Models

\begin{tabular}{|c|c|c|c|}
\hline Variables & Risk models & per at risk & expected risk of death \\
\hline \multirow[t]{2}{*}{ Age of diagnosis } & $<20$ years & 7 & 0.57 \\
\hline & $\geq 20$ years old & 20705 & 0.45 \\
\hline \multirow[t]{2}{*}{ Sex } & Female & 13109 & 0.38 \\
\hline & Male & 7603 & 0.56 \\
\hline \multirow[t]{2}{*}{ Site rec with Kaposi and mesothelioma } & Lung and bronchus & 9472 & 0.53 \\
\hline & Others & 11240 & 0.38 \\
\hline \multirow[t]{5}{*}{ Grade } & Well differentiated; Grade I & 790 & 0.13 \\
\hline & Moderately differentiated; Grade II & 4017 & 0.32 \\
\hline & Poorly differentiated; Grade III & 8793 & 0.48 \\
\hline & Undifferentiated; anaplastic; Grade IV & 780 & 0.48 \\
\hline & Unknown & 6332 & 0.52 \\
\hline \multirow[t]{4}{*}{ SEER Staging } & Localized & 6801 & 0.19 \\
\hline & Regional & 6196 & 0.45 \\
\hline & Distant & 4836 & 0.70 \\
\hline & Unstaged/ohters & 2879 & 0.61 \\
\hline \multirow[t]{4}{*}{ Rural-Urban Continuum Code 2003} & $\begin{array}{l}\text { Counties in metropolitan areas ge } 1 \text { mill } \\
250,000 \text { to } 1 \text { million pop/Urban pop of } \mathrm{ge}\end{array}$ & $\begin{array}{l}\text { ion pop/ Cc } \\
20,000 \mathrm{ad}\end{array}$ & $\begin{array}{l}\text { in metropolitan areas of } \\
\text { o a metropolitan }\end{array}$ \\
\hline & area & 18446 & 0.44 \\
\hline & versus & & \\
\hline & Others & 2266 & 0.49 \\
\hline \multirow[t]{2}{*}{ County Family Income } & $\geq \$ 50000$ & 11952 & 0.44 \\
\hline & $<\$ 50000$ & 8760 & 0.46 \\
\hline \multirow[t]{2}{*}{ County $\%$ college graduate } & $\geq 25$ college graduate & 10557 & 0.45 \\
\hline & $<25 \%$ college graduate & 10155 & 0.45 \\
\hline \multirow[t]{2}{*}{ Race } & White/others & 18634 & 0.44 \\
\hline & Black & 2078 & 0.54 \\
\hline \multirow[t]{2}{*}{ Radiation treatment given } & Beam radiation & 6725 & 0.56 \\
\hline & Others & 13987 & 0.39 \\
\hline \multirow[t]{2}{*}{ Reason no cancer-directed surgery } & Surgery performed & 13639 & 0.34 \\
\hline & Others & 7073 & 0.66 \\
\hline
\end{tabular}


Analysis of SEER Adenosquamous Carcinoma Data for Cause Specific Survival Predictors and Socioeconomic Disparities

Table 3. The Site Distribution of Adenosquamous Cancers

\begin{tabular}{|c|c|c|}
\hline Site & Jumber & Percentage \\
\hline Lung and Bronchus & 9472 & 45.72973495 \\
\hline Miscellaneous & 379 & 1.829768744 \\
\hline Corpus Uteri & 4221 & 20.37850625 \\
\hline Breast & 213 & 1.02833969 \\
\hline Cervix Uteri & 2813 & 13.58084295 \\
\hline Pancreas & 656 & 3.16709313 \\
\hline Other Non-Epithelial Skin & 137 & 0.661420364 \\
\hline Rectum & 113 & 0.545551103 \\
\hline Stomach & 361 & 1.742866799 \\
\hline Gallbladder & 436 & 2.104958239 \\
\hline Esophagus & 512 & 2.471877565 \\
\hline Other Biliary & 75 & 0.36209144 \\
\hline Nose, Nasal Cavity and Middle Ear & 33 & 0.159320234 \\
\hline Kidney and Renal Pelvis & 12 & 0.05793463 \\
\hline Salivary Gland & 78 & 0.376575098 \\
\hline Other Female Genital Organs & 34 & 0.16414812 \\
\hline Cecum & 87 & 0.420026071 \\
\hline Larynx & 61 & 0.294501038 \\
\hline Urinary Bladder & 58 & 0.28001738 \\
\hline Small Intestine & 17 & 0.08207406 \\
\hline Transverse Colon & 28 & 0.135180804 \\
\hline Ovary & 243 & 1.173176266 \\
\hline Anus, Anal Canal and Anorectum & 82 & 0.395886641 \\
\hline Ascending Colon & 51 & 0.246222179 \\
\hline Splenic Flexure & 10 & 0.048278859 \\
\hline Uterus, NOS & 42 & 0.202771206 \\
\hline Descending Colon & 10 & 0.048278859 \\
\hline Sigmoid Colon & 65 & 0.313812581 \\
\hline Vulva & 28 & 0.135180804 \\
\hline Prostate & 35 & 0.168976005 \\
\hline Vagina & 70 & 0.337952011 \\
\hline Tonsil & 22 & 0.106213489 \\
\hline Rectosigmoid Junction & 35 & 0.168976005 \\
\hline Gum and Other Mouth & 34 & 0.16414812 \\
\hline Hypopharynx & 23 & 0.111041375 \\
\hline Floor of Mouth & 14 & 0.067590402 \\
\hline Tongue & 43 & 0.207599092 \\
\hline \multicolumn{3}{|c|}{ Trachea, Mediastinum and Other Respiratory Organs } \\
\hline & 10 & 0.048278859 \\
\hline Penis & 3 & 0.014483658 \\
\hline Thyroid & 5 & 0.024139429 \\
\hline Oropharynx & 12 & 0.05793463 \\
\hline Other Urinary Organs & 11 & 0.053106745 \\
\hline Other Digestive Organs & 7 & 0.033795201 \\
\hline Lip & 2 & 0.009655772 \\
\hline Eye and Orbit & 2 & 0.009655772 \\
\hline Peritoneum, Omentum and Mesentery & y 4 & 0.019311543 \\
\hline Other Oral Cavity and Pharynx & 5 & 0.024139429 \\
\hline Liver & 7 & 0.033795201 \\
\hline Hepatic Flexure & 14 & 0.067590402 \\
\hline Nasopharynx & 8 & 0.038623087 \\
\hline Ureter & 4 & 0.019311543 \\
\hline Large Intestine, NOS & 7 & 0.033795201 \\
\hline Intrahepatic Bile Duct & 4 & 0.019311543 \\
\hline Other Endocrine including Thymus & 1 & 0.004827886 \\
\hline Appendix & 2 & 0.009655772 \\
\hline Soft Tissue including Heart & 1 & 0.004827886 \\
\hline
\end{tabular}

from children with cancer. Pediatr Blood Cancer, 56, 9941002.

Cheung MC, Zhuge Y, Yang R, et al (2010). Incidence and outcomes of extremity soft-tissue sarcomas in children. $J$ Surg Res, 163, 282-9.
Cheung MR (2014a). Optimization of predictors of Ewing sarcoma cause-specific survival: a population study. Asian Pac J Cancer Prev, 15, 4143-5.

Cheung MR (2014b). Receiver operating characteristic curve analysis of SEER medulloblastoma and primitive neuroectodermal tumor (PNET) outcome data: identification and optimization of predictive models. Asian Pac J Cancer Prev, 15, 6781-5.

Cheung MR (2014c). Surveying and optimizing the predictors for ependymoma specific survival using SEER data. Asian Pac J Cancer Prev, 15, 867-70.

Cheung MR (2014d). Under-use of radiotherapy in stage III bronchioaveolar lung cancer and socio-economic disparities in cause specific survival: a population study. Asian Pac J Cancer Prev, 15, 4091-4.

Cheung R (2014e). Epidemiology and radiotherapy of hepatocellular carcinoma. Int J Cancer Clin Res, $1,1$.

Cheung R (2015a). Smoking, air pollution and cancer: global epidemiology, public health and genomics. Ann Transl Med Epidemiol, 2, 1-7.

Cheung R 2015b. Topics on radiotherapy, global cancer epidemiology and public health. Lambert Academic Publishing.

Cheung R 2015 (In press). Determining best contours in radiotherapy treatment in a modern era, and asian American medical epidemiology: public health point of view.

Cheung R 2015, in press. Contemporary notes on public health and radiotherapy topics, Austin Publishing Group, Austin eBooks.

Cheung R, Altschuler MD, D'Amico AV, et al (2001a). ROCoptimization may improve risk stratification of prostate cancer patients. Urology, 57, 286-90.

Cheung R, Altschuler MD, D'Amico AV, et al (2001b). Using the receiver operator characteristic curve to select pretreatment and pathologic predictors for early and late post-prostatectomy PSA failure. Urology, 58, 400-5.

D'Amico AV, Desjardin A, Chung A, et al (1998). Assessment of outcome prediction models for patients with localized prostate carcinoma managed with radical prostatectomy or external beam radiation therapy. Cancer, 82, 1887-96.

Galic V, Herzog TJ, Lewin SN, et al (2012). Prognostic significance of adenocarcinoma histology in women with cervical cancer. Gynecol Oncol, 125, 287-91.

Hanley JA, McNeil BJ (1982). The meaning and use of the area under a receiver operating characteristic (ROC) curve. Radiology, 143, 29-36.

Marcus DM, Goodman M, Jani AB, et al (2012). Acomprehensive review of incidence and survival in patients with rare histological variants of prostate cancer in the United States from 1973 to 2008. Prostate Cancer Prostatic Dis, 15, 283-8.

Masoomi H, Ziogas A, Lin BS, et al (2012). Population-based evaluation of adenosquamous carcinoma of the colon and rectum. Dis Colon Rectum, 55, 509-14.

McDowell HP, Foot AB, Ellershaw C, et al (2010). Outcomes in paediatric metastatic rhabdomyosarcoma: results of The International Society of Paediatric Oncology (SIOP) study MMT-98. Eur J Cancer, 46, 1588-95.

Ognjanovic S, Linabery AM, Charbonneau B, et al (2009). Trends in childhood rhabdomyosarcoma incidence and survival in the United States, 1975-2005. Cancer, 115, 4218-26.

Pappo AS, Krailo M, Chen Z, et al (2010). Infrequent tumor initiative of the Children's Oncology Group: initial lessons learned and their impact on future plans. J Clin Oncol, 28, 5011-6.

Perez EA, Kassira N, Cheung MC, et al (2011). Rhabdomyosarcoma in children: a SEER population based 


\section{Rex Cheung}

study. J Surg Res, 170, e243-51.

Sultan I, Qaddoumi I, Yaser S, et al (2009). Comparing adult and pediatric rhabdomyosarcoma in the surveillance, epidemiology and end results program, 1973 to 2005: an analysis of 2,600 patients. J Clin Oncol, 27, 3391-7.

Travis WD, Lubin J, Ries L, et al (1996). United States lung carcinoma incidence trends: declining for most histologic types among males, increasing among females. Cancer, 77, 2464-70.

Wang J, Wang FW, Lagrange CA, et al (2010). Clinical features and outcomes of 25 patients with primary adenosquamous cell carcinoma of the prostate. Rare Tumors, $2,47$. 